\title{
Can I Stop Anticoagulation When Cancer Is in Remission?
}

\author{
Norimichi Koitabashi, MD, PhD
}

I $\mathrm{t}$ has been more than 150 years since the association between cancer and thrombosis was reported by Bouillaud and Trousseau. ${ }^{1}$ Cancer cells activate the coagulation-fibrinolysis and platelet systems through various pathways during their growth and invasion. ${ }^{\mathbf{1 , 2}}$ Consequently, cancer patients frequently suffer from venous or arterial thrombosis, which has a significant effect on their prognosis. In recent years, thrombosis in cancer patients has been termed cancer-associated thrombosis (CAT), and has become an important topic of investigation in Oncocardiology/Cardio-oncology. ${ }^{1}$

Venous thromboembolism (VTE) is caused by a combination of factors, known as Virchow's triad: hypercoagulability, stasis of venous blood flow, and endothelial damage of various degrees. ${ }^{1}$ Cancer cells produce tissue factors and various pro-coagulants that activate the coagulation system.1,2 In addition, cancer patients are often inactive, which, in combination with vascular compression by the tumor and lymph nodes, can lead to venous stasis. Furthermore, cancer surgery, anticancer drugs and central venous catheters can damage the venous endothelium. In Japan, cancer is the most common cause of VTE, followed by VTE of unknown etiology. ${ }^{3}$ Although treatment guidelines recommend long-term anticoagulation for cancer-related VTE, cancer patients are also at a high risk for bleeding, making continued anticoagulation a major dilemma.,45

Worldwide, low-molecular-weight (LMW) heparin has been used for the treatment of VTE in CAT, ${ }^{4}$ but based on the results of recent large-scale clinical studies, direct oral anticoagulants (DOACs) are now being used in cancer cases as well, ${ }^{6,7}$ On the other hand, in Japan, the use of LMW heparin is not approved by national insurance as a treatment for VTE, and DOACs or vitamin $\mathrm{K}$ antagonists (VKA; warfarin) are mainly used for cancer-related VTE. ${ }^{8}$ Although a number of clinical studies have been reported worldwide on long-term anticoagulation in cancer patients and the pros and cons of discontinuing LMW heparin, ${ }^{9}$ providing a rationale for the current treatment strategy, there is little evidence on the use of DOACs for very longterm treatment of VTE in cancer patients.5,6

In this issue of the Journal, Hara et $\mathbf{a l}^{\mathbf{1 0}}$ report a singlecenter retrospective analysis for a composite outcome of all-cause death, VTE recurrence, bleeding and a composite outcome of VTE-related death, recurrence and bleeding using clinical data from patients with VTE. A total of 893 patients treated for acute VTE between 2011 and 2019

Article p????

were reviewed in detail for their background factors, treatments and prognoses. Cancer-related VTE accounted for 343 of the cases $(38.4 \%)$. A unique aspect of this study was analysis of the "cancer remission group". The primary endpoint, a composite of all-cause death, VTE recurrence and bleeding complications, was significantly more common in the continued cancer treatment group compared with the other groups at up to 3 years of follow-up, although the "cancer remission group" had a very good prognosis. ${ }^{10}$ Most of the previous clinical studies of VTE in CAT have examined prognosis within 1 year, ${ }^{5}$ and very few studies have compared prognosis for more than 3 years. ${ }^{8}$ In addition, this cohort study compared the efficacy of VTE treatment with DOACs and VKA. The results showed that the use of DOACs was associated with significantly fewer bleeding events compared with VKA in the continuous cancer treatment group, making DOACs a safer anticoagulant therapy during cancer treatment.

Although this was a single-center retrospective study with a small number of patients and needs to be followed up on a larger scale, it does provide some important realworld information. The first point is that once cancer treatment is completed, even if anticoagulation is discontinued, the risk of recurrence of VTE is similar to that of patients who had VTE as a temporary risk. In Japan, it has become common practice to provide anticoagulation as permanently as possible for VTE patients with a history of cancer. Sakamoto et al reported that very long-term (up to 3 years) anticoagulation was used in nearly half of their patients with cancer-related VTE. ${ }^{8}$ However, the need for anticoagulation should be determined by balancing the risk of thrombosis and bleeding. ${ }^{69}$ Cancer treatment has made remarkable progress in recent years and an increasing number of patients diagnosed with cancer are expected to have a long-term prognosis of life. ${ }^{11}$ If the cancer is judged to be in remission following treatment, anticoagulation should be discontinued. However, careful follow-up is necessary for cancer recurrence. If the cancer recurs, the risk of VTE recurrence also increases, in which case prophylactic anticoagulants might be necessary during further cancer treatment. 5

Another observation of this study is that DOACs reduced the risk of bleeding in the VTE subgroup of the "continued

The opinions expressed in this article are not necessarily those of the editors or of the Japanese Circulation Society.

Received October 15, 2021; accepted October 15, 2021; J-STAGE Advance Publication released online November 13, 2021

Department of Cardiovascular Medicine, Gunma University Graduate School of Medicine, Maebashi, Japan

Mailing address: Norimichi Koitabashi, MD, PhD, Department of Cardiovascular Medicine, Gunma University Graduate School of Medicine, 3-39-22 Showa-machi, Maebashi 371-8511, Japan. E-mail: koitabas@gunma-u.ac.jp

All rights are reserved to the Japanese Circulation Society. For permissions, please e-mail: cj@j-circ.or.jp

ISSN-1346-9843 
cancer treatment group" compared with VKA. ${ }^{10}$ The use of an inferior vena cava filter (IVCF) was more common in the VKA group in this study, although this does not seem to have affected the results, because it has been reported in Japan that the use of IVCF does not necessarily have a negative effect on prognosis. ${ }^{12}$ However, the proportion of cancer patients in this cohort who were given edoxaban was high, and because, unlike other DOACs, the dose of edoxaban for VTE is adjusted according to body weight and renal function, ${ }^{7}$ the high proportion of edoxaban usage might have been responsible for the reduced bleeding risk observed in this study. However, there was no significant difference in the incidence of bleeding events between edoxaban and other DOACs. ${ }^{10}$ In this study, patient prognosis in the cancer treatment group was worse when anticoagulation was discontinued due to bleeding as compared with when it was discontinued for other reasons. ${ }^{10}$ However, evaluation of the indications for anticoagulation should consider the balance between bleeding risk and thrombosis risk. ${ }^{6}$ In this study, the sample size was insufficient to conclude that anticoagulation should be continued even if bleeding occurs.

Because the reason for discontinuing anticoagulation is left to the physician's judgment, selection bias is a significant factor in the results of this study. VKA and DOACs differ greatly in terms of interactions with other drugs ${ }^{7}$ and their cost, which limits a retrospective analysis on this scale. Larger studies and prospective studies are needed to confirm the validity of these results. As adherence to DOACs is expected to improve compared with that to subcutaneous LMW heparin or VKAs, ${ }^{13}$ and because very long-term anticoagulation with DOACs has become easier, ${ }^{14}$ this article should be remembered as an important source of information when considering the indications for very long-term or permanent anticoagulation for cancerrelated VTE and the decision to discontinue it.

\section{Financial / Nonfinancial Disclosures}

N.K. received lecture fees from Bayer Yakuhin.

\section{References}

1. Mukai M, Oka T. Mechanism and management of cancer-associated thrombosis. J Cardiol 2018; 72: 89-93.

2. Varki A. Trousseau's syndrome: Multiple definitions and multiple mechanisms. Blood 2007; 110: 1723-1729.

3. Nakamura M, Miyata T, Ozeki Y, Takayama M, Komori K, Yamada N, et al. Current venous thromboembolism management and outcomes in Japan. Circ J 2014; 78: 708-717.

4. Kearon C, Akl EA, Ornelas J, Blaivas A, Jimenez D, Bounameaux $\mathrm{H}$, et al. Antithrombotic therapy for VTE disease: Chest guideline and expert panel report. Chest 2016; 149: 315-352.

5. Marin-Romero S, Jara-Palomares L. Extended treatment of cancer-associated thrombosis. Thromb Res 2019; 181: 1-9.

6. Khan F, Tritschler T, Kahn SR, Rodger MA. Venous thromboembolism. Lancet 2021; 398: 64-77.

7. Chan N, Sobieraj-Teague M, Eikelboom JW. Direct oral anticoagulants: Evidence and unresolved issues. Lancet 2020; 396: $1767-1776$.

8. Sakamoto J, Yamashita Y, Morimoto T, Amano H, Takase T, Hiramori S, et al. Cancer-associated venous thromboembolism in the real world From the COMMAND VTE registry. Circ $J$ 2019; 83: 2271-2281.

9. Agnelli G, Becattini C. Treatment of DVT: How long is enough and how do you predict recurrence. J Thromb Thrombolysis 2008; 25: $37-44$.

10. Hara N, Lee T, Nozato T, Matsuyama MT, Okata S, Nagase M, et al. Effectiveness and safety of direct oral anticoagulants vs. warfarin and recurrence after discontinuation in patients with acute venous thromboembolism in the real world. Circ $J$, doi:10.1253/circj.CJ-21-0588.

11. Helmink BA, Gaudreau PO, Wargo JA. Immune checkpoint blockade across the cancer care continuum. Immunity 2018; 48: $1077-1080$.

12. Ohyama Y, Koitabashi N, Nakamura T, Sumita Y, Nakai M, Nishimura K, et al. Effect of inferior vena cava filter on venous thromboembolism mortality in Japan: JROAD and JROADDPC registry analysis. Circ Rep 2019; 1: 296-302.

13. Khorana AA, McCrae KR, Milentijevic D, Fortier J, Nelson WW, Laliberte F, et al. Current practice patterns and patient persistence with anticoagulant treatments for cancer-associated thrombosis. Res Pract Thromb Haemost 2017; 1: 14-22.

14. Weitz JI, Lensing AWA, Prins MH, Bauersachs R, BeyerWestendorf J, Bounameaux $\mathrm{H}$, et al. Rivaroxaban or aspirin for extended treatment of venous thromboembolism. $N$ Engl J Med 2017; 376: $1211-1222$. 